\title{
A Machine-Based Approach to Preoperatively Identify Patients with the Most and Least Benefit Associated with Resection for Intrahepatic Cholangiocarcinoma: An International Multi-institutional Analysis of 1146 Patients
}

Diamantis I. Tsilimigras, MD ${ }^{1}$, Rittal Mehta, MS ${ }^{1}$, Dimitrios Moris, MD, PhD ${ }^{1}$, Kota Sahara, MD ${ }^{1}$, Fabio Bagante, MD ${ }^{1}$, Anghela Z. Paredes, MD, MS ${ }^{1}$, Amika Moro, $\mathbf{M D}^{1}$, Alfredo Guglielmi, $\mathbf{M D}^{2}$, Luca Aldrighetti, $\mathrm{MD}^{3}$, Matthew Weiss, $\mathrm{MD}^{4}$, Todd W. Bauer, $\mathrm{MD}^{5}$, Sorin Alexandrescu, $\mathrm{MD}^{6}$, George A. Poultsides, $\mathrm{MD}^{7}$, Shishir K. Maithel, $\mathrm{MD}^{\mathbf{8}}$, Hugo P. Marques, MD ${ }^{9}$, Guillaume Martel, $\mathbf{M D}^{10}$, Carlo Pulitano, $\mathrm{MD}^{11}$, Feng Shen, $\mathrm{MD}^{12}$, Olivier Soubrane, $\mathrm{MD}^{13}$, Bas Groot Koerkamp, $\mathbf{M D}^{14}$, Itaru Endo, MD, PhD ${ }^{15}$, and Timothy M. Pawlik, MD, MPH, PhD, FACS, FRACS (Hon.) ${ }^{1,16}$ (i)

${ }^{1}$ Division of Surgical Oncology, Department of Surgery, Wexner Medical Center and James Comprehensive Cancer Center, The Ohio State University, Columbus, $\mathrm{OH} ;{ }^{2}$ Department of Surgery, University of Verona, Verona, Italy; ${ }^{3}$ Department of Surgery, Ospedale San Raffaele, Milan, Italy; ${ }^{4}$ Department of Surgery, Johns Hopkins Hospital, Baltimore, MD; ${ }^{5}$ Department of Surgery, University of Virginia, Charlottesville, VA; ${ }^{6}$ Department of Surgery, Fundeni Clinical Institute, Bucharest, Romania; ${ }^{7}$ Department of Surgery, Stanford University, Stanford, CA; ${ }^{8}$ Department of Surgery, Emory University, Atlanta, GA; ${ }^{9}$ Department of Surgery, Curry Cabral Hospital, Lisbon, Portugal; ${ }^{10}$ Department of Surgery, University of Ottawa, Ottawa, Canada; ${ }^{11}$ Department of Surgery, Royal Prince Alfred Hospital, University of Sydney, Sydney, Australia; ${ }^{12}$ Department of Surgery, Eastern Hepatobiliary Surgery Hospital, Shanghai, China;

${ }^{13}$ Department of Hepatobiliopancreatic Surgery and Liver Transplantation, AP-HP, Beaujon Hospital, Clichy, France;

${ }^{14}$ Department of Surgery, Erasmus University Medical Centre, Rotterdam, The Netherlands; ${ }^{15}$ Department of Gastroenterological Surgery, Yokohama City University School of Medicine, Yokohama, Japan; ${ }^{16}$ Department of Surgery, The Urban Meyer III and Shelley Meyer Chair for Cancer Research, Wexner Medical Center, The Ohio State University, Columbus, $\mathrm{OH}$

\begin{abstract}
Background. Accurate risk stratification and patient selection is necessary to identify patients who will benefit the most from surgery or be better treated with other nonsurgical treatment strategies. We sought to identify which patients in the preoperative setting would likely derive the
\end{abstract}

Electronic supplementary material The online version of this article (https://doi.org/10.1245/s10434-019-08067-3) contains supplementary material, which is available to authorized users.

(C) Society of Surgical Oncology 2019

First Received: 29 August 2019; Published Online: 14 November 2019

T. M. Pawlik, MD, MPH, PhD, FACS, FRACS (Hon.)

e-mail: tim.pawlik@osumc.edu most or least benefit from resection of intrahepatic cholangiocarcinoma (ICC).

Methods. Patients who underwent curative-intent resection for ICC between 1990 and 2017 were identified from an international multi-institutional database. A machinebased classification and regression tree (CART) was used to generate homogeneous groups of patients relative to overall survival (OS) based on preoperative factors.

Results. Among 1146 patients, CART analysis revealed tumor number and size, albumin-bilirubin (ALBI) grade and preoperative lymph node (LN) status as the strongest prognostic factors associated with OS among patients undergoing resection for ICC. In turn, four groups of patients with distinct outcomes were generated through machine learning: Group $1(n=228)$ : single ICC, size $\leq 5 \mathrm{~cm}$, ALBI grade I, negative preoperative LN status; Group $2(n=708)$ : (1) single tumor $>5 \mathrm{~cm}$, (2) single tumor $\leq 5 \mathrm{~cm}$, ALBI grade $2 / 3$, and (3) single tumor 
$\leq 5 \mathrm{~cm}$, ALBI grade 1, metastatic/suspicious LNs; Group 3 (n=150): $2-3$ tumors; Group $4(n=60): \geq 4$ tumors. 5 -year OS among Group 1, 2, 3, and 4 patients was $60.5 \%$, $35.8 \%, 27.5 \%$, and $3.8 \%$, respectively $(p<0.001)$. Similarly, 5-year disease-free survival (DFS) among Group 1, 2 , 3 , and 4 patients was $47 \%, 27.2 \%, 6.8 \%$, and $0 \%$, respectively $(p<0.001)$.

Conclusions. The machine-based CART model identified distinct prognostic groups of patients with distinct outcomes based on preoperative factors. Survival decision trees may be useful as guides in preoperative patient selection and risk stratification.

While a relatively rare cancer, the incidence of intrahepatic cholangiocarcinoma (ICC) has increased worldwide almost threefold over the past three decades. ${ }^{1,2}$ Surgery remains the mainstay of treatment and the only chance for long-term survival among patients diagnosed with ICC. Despite technologic advances and improvements in surgical techniques, prognosis of patients with ICC still remains dismal with 5-year overall survival (OS) ranging from 20 to $40 \%$ after curative-intent resection. ${ }^{3,4}$ In addition, only one in four patients achieve an optimal textbook outcome (i.e., R0 resection, no perioperative transfusion, no postoperative surgical complications, no prolonged length of stay, no 30-day readmission, and no 30-day mortality) following surgery for ICC, as many patients experience some type of complication related to surgery. ${ }^{5}$ Furthermore, the majority of patients recur within 2 years of surgery, thereby compromising any chance of long-term survival. ${ }^{6}$ As such, patient selection and risk stratification to identify patients who may be the optimal candidates for surgery has particular importance for patients with ICC.

Given the general poor prognosis of patients with ICC, some clinicians have proposed that patients with ICC should receive neoadjuvant therapy before surgery or even be treated with other nonsurgical treatment modalities. ${ }^{7}$ In turn, several prognostic tools have been developed to identify patients at risk for adverse outcomes following ICC resection to help inform preoperative decisions around treatment. ${ }^{8-10}$ The majority of these tools have relied, however, on pathological data, which prevents applicability in the preoperative setting. ${ }^{8-10}$ To this end, Sasaki et al. ${ }^{11}$ proposed a preoperative risk score that consisted of radiologic tumor size and serum biomarkers, including cancer antigen (CA)19-9, neutrophil-to-lymphocyte ratio, and albumin, to predict prognosis following resection of ICC. In addition, our group recently reported that albuminbilirubin (ALBI) grade-a marker of underlying liver function reserve-also was an important predictor of both short- and long-term outcomes among patients undergoing surgery for ICC. ${ }^{12}$ Nevertheless, models based on standard statistical techniques have been criticized for the discordance in the predicted versus actual postoperative outcomes, suggesting that preoperative patient risk stratification based on these models may be problematic and sometimes misleading. ${ }^{13}$

Survival tree modelling is a machine-learning statistical technique that recently has been used in health care increasingly as a means to aid in treatment decision making. ${ }^{14,15}$ Proponents of machine learning include an unsupervised approach with minimal human intervention in the model construction, thereby limiting bias and error. In particular, machine learning is adept at reviewing large volumes of data and identifying patterns and trends that might not be apparent to a human. In addition, machine learning may identify causal relationships that may not have been evident with other techniques. Because machine learning technology typically improves efficiency and accuracy over time as increasing amounts of data are processed, models based on this approach typically have improved predictive accuracy. ${ }^{14,15}$ As such, the objective of the current study was to identify preoperative factors that mattered the most in terms of survival following surgery for ICC. Specifically, we sought to identify groups of patients with distinct outcomes and characterize patients who derived the most or least benefit from surgery using a machine learning approach.

\section{METHODS}

\section{Study Population and Inclusion Criteria}

Patients who underwent curative-intent liver resection for ICC between January 1990 and December 2017 were identified using a multi-institutional database. Patients underwent surgery in one of the following tertiary institutions that made up the International Intrahepatic Cholangiocarcinoma Study Group; The Ohio State University Comprehensive Cancer Center, Columbus, OH; Johns Hopkins Hospital, Baltimore, MD; Stanford University, Stanford, CA; University of Virginia, Charlottesville, VS; Winship Cancer Institute, Emory University, Atlanta, GA; Fundeni Clinical Institute, Bucharest, Romania; Scientific Institute San Raffaele, Vita-Salute San Raffaele University, Milan, Italy; University of Verona, Verona, Italy; Curry Cabral Hospital, Lisbon, Portugal; Beaujon Hospital, Clichy, France; Erasmus University Medical Centre, Rotterdam, Netherlands; University of Ottawa, Ontario, Canada; Eastern Hepatobiliary Surgery Hospital, Shanghai, China; Yokohama City University School of Medicine, Yokohama, Japan; Royal Prince Alfred Hospital, University of Sydney, Sydney, Australia. Patients who did not undergo curative-intent 
resection, had concurrent extrahepatic disease at the time of hepatectomy or had missing follow-up data were excluded. The Institutional Review Board of all participating institutions approved this study.

\section{Variables of Interest and Definitions}

Demographic and clinicopathologic data included age, sex, American Society of Anesthesiologists (ASA) score, preoperative serum CA19-9, and carcinoembryonic antigen (CEA) levels, ALBI grade, presence of cirrhosis, type of resection (i.e., minor or major), tumor location (i.e., unifocal or multifocal), preoperative lymph node (LN) status (i.e., negative, suspicious, or positive LNs) and tumor size, pathologic $\mathrm{T}$ and $\mathrm{N}$ stage, margin status (i.e., R0, R1, or R2 resection), morphological type (i.e., MF: mass-forming; IG: intraductal growth; or PI: periductal infiltrating), tumor grade, presence of major vascular or micro-vascular invasion, as well as receipt of adjuvant chemotherapy.

Pathologic tumor T- and N-stage were defined according to the American Joint Committee on Cancer (AJCC) 8th edition staging manual. ${ }^{16}$ Major hepatectomy was defined as the resection of three or more Couinaud segments. ${ }^{17}$ Major vascular invasion was defined as invasion of the first- and second-order branches of the portal vein or hepatic arteries or as invasion of one or more of the three hepatic veins, whereas microvascular invasion was defined as intraparenchymal vascular involvement identified on histological examination. ${ }^{16}$ Furthermore, the ALBI score was calculated using the following formula: $\left[\log _{10}\right.$ bilirubin $(\mu \mathrm{mol} / \mathrm{L}) \times 0.66]+[$ albumin $(\mathrm{g} / \mathrm{L}) \times-0.085]$, and patients were categorized into three groups as follows: grade $1 \leq-2.60$, grade $2 \geq-2.60$ and $\leq-1.39$, and grade $3 \geq-1.39 .{ }^{12,18}$ Information on whether multiple tumors represented satellite lesions, intrahepatic metastases, or multiple primary tumors was not available; as such, location of tumor (i.e., unilobar, bilobar) was used as a surrogate for the purposes of subgroup analysis.

\section{Statistical Analysis}

Continuous and categorical variables were presented as median [interquartile range (IQR)] and frequency (\%), respectively. Differences in baseline characteristics were assessed using Wilcoxon rank-sum test for continuous and Chi squared test or Fisher's exact test for categorical variables, as appropriate. Overall survival (OS) defined as the time interval between the date of hepatectomy for ICC and the date of death or last follow-up was the primary outcome of the study. Disease-free survival (DFS) was defined as the time interval between the date of hepatectomy and the date of recurrence or last follow-up. Differences in survival among groups were assessed by
Kaplan-Meier curves and log-rank test. Statistical significance was determined at $\alpha=0.05$. Among patients with multifocal tumors, bivariate, and multivariable cox regression analyses were performed to assess for factors associated with survival. Variables significant on bivariate analysis $(p<0.05)$ were entered into the multivariable model. A nonparametric risk prediction model, known as Classification and Regression Tree (CART) model, was used to generate homogeneous groups of patients relative to OS based on preoperative factors, as previously described. ${ }^{14,19}$ Candidate variables for the survival tree analysis were limited to those that could be known preoperatively, including preoperative tumor size and number, as well as age, gender, ASA class, serum CA 19-9 and CEA levels, ALBI grade, and preoperative LN status. Missing data were handled with the use of surrogate splits, which is the standard way to handle missing data with partykit package. ${ }^{20,21}$ In order to reduce overfitting while building the survival tree, pruning in the training data was performed using minimum Cook's D parameter. To assess the possibility of overfitting, ten-fold cross validation was used. To assess the predictive performance of the final model, the c-index for time-to-event data was calculated with the bootstrapping resample method $(n=2000)$ using the $\mathrm{R}$ CRAN package ipred. All statistical analyses were performed with the SPSS, version 25 statistical package (IBM Corp. Armonk, NY) and R CRAN software for statistical computing v. 3.6.0 with the additional packages: survival, partykit, rpart, Hmisc, caret, ipred, and ROCR.

\section{RESULTS}

\section{Demographics of the Analytic Cohort}

A total of 1146 patients underwent curative-intent resection for ICC and were included in the final analytic cohort (Table 1). Median patient age was 60.4 years (interquartile range [IQR]: 51.7-69.1) and more than half of patients $(n=638,55.6 \%)$ were male. Median preoperative CA19-9 and CEA levels were $49.3 \mathrm{UI} / \mathrm{mL}$ (IQR: 16.9-211.5) and $2.4 \mathrm{ng} / \mathrm{L}$ (IQR: 1.4-4.3), respectively. Overall, 487 (64.1\%) patients had ALBI grade 1, whereas $273(35.9 \%)$ had ALBI grade 2 or 3 . The majority of patients had unifocal disease $(n=844,81.6 \%)$ and underwent a major liver resection $(n=626,60.9 \%)$. Median tumor size was $6.0 \mathrm{~cm}$ (IQR: 4.0-8.4). On pathology, $45.8 \%(n=521)$ of patients had T1a/T1b disease, whereas $27.2 \%(n=284)$ and $17.0 \%(n=177)$ had $\mathrm{N} 0$ and N1 disease, respectively. Most patients had an R0 resection $(n=974,86.2 \%)$ and MF or IG ICC type ( $n=935,86.9 \%)$, whereas only a small subset of individuals had major vascular invasion $(n=157,13.9 \%)$. 
TABLE 1 Clinicopathologic features of patients $(N=1146)$

\begin{tabular}{|c|c|}
\hline Variable & Total, $N(\%)$ \\
\hline Age, median (IQR) & $60.4(51.7,69.1)$ \\
\hline Male & $638(55.6)$ \\
\hline \multicolumn{2}{|l|}{ ASA } \\
\hline$\leq 2$ & $632(66.9)$ \\
\hline$>2$ & $312(33.1)$ \\
\hline CA19-9, UI/mL, median (IQR) & $49.3(16.9,211.5)$ \\
\hline CEA, ng/L, median (IQR) & $2.4(1.4,4.3)$ \\
\hline \multicolumn{2}{|l|}{ ALBI grade } \\
\hline 1 & $487(64.1)$ \\
\hline $2 / 3$ & $273(35.9)$ \\
\hline Cirrhosis & $123(10.8)$ \\
\hline \multicolumn{2}{|l|}{ Type of resection } \\
\hline Minor resection & $402(39.1)$ \\
\hline Major resection & $626(60.9)$ \\
\hline \multicolumn{2}{|l|}{ Location } \\
\hline Unifocal & $844(81.6)$ \\
\hline Multifocal & $190(18.4)$ \\
\hline \multicolumn{2}{|l|}{ Preoperative LN status } \\
\hline Negative & $720(78.5)$ \\
\hline Suspicious & $137(14.9)$ \\
\hline Positive & $60(6.5)$ \\
\hline Tumor size $(\mathrm{cm})$, median (IQR) & $6.0(4.0,8.4)$ \\
\hline \multicolumn{2}{|l|}{ AJCC 8th edition T stage } \\
\hline $\mathrm{T} 1 \mathrm{a} / \mathrm{T} 1 \mathrm{~b}$ & $521(45.8)$ \\
\hline $\mathrm{T} 2 / \mathrm{T} 3 / \mathrm{T} 4$ & $617(54.2)$ \\
\hline \multicolumn{2}{|l|}{ AJCC 8th edition $\mathrm{N}$ stage } \\
\hline $\mathrm{Nx}$ & $583(55.8)$ \\
\hline No & $284(27.2)$ \\
\hline N1 & $177(17.0)$ \\
\hline \multicolumn{2}{|l|}{ Margin status } \\
\hline R0 & $974(86.2)$ \\
\hline $\mathrm{R} 1$ & $147(13.0)$ \\
\hline $\mathrm{R} 2$ & $9(0.8)$ \\
\hline \multicolumn{2}{|l|}{ Morphologic type } \\
\hline MF, IG & $935(86.9)$ \\
\hline $\mathrm{PI}, \mathrm{MF}+\mathrm{PI}$ & $141(13.1)$ \\
\hline \multicolumn{2}{|l|}{ Grade } \\
\hline Well to moderate & $878(82.4)$ \\
\hline Poor to undifferentiated & $188(17.6)$ \\
\hline \multicolumn{2}{|l|}{ Major vascular invasion } \\
\hline No & $972(86.1)$ \\
\hline Yes & $157(13.9)$ \\
\hline \multicolumn{2}{|l|}{ Microvascular invasion } \\
\hline No & $764(68.3)$ \\
\hline Yes & $354(31.7)$ \\
\hline \multicolumn{2}{|l|}{ Adjuvant chemotherapy } \\
\hline No & $749(68.7)$ \\
\hline Yes & $342(31.3)$ \\
\hline
\end{tabular}

TABLE 1 continued

\begin{tabular}{lc}
\hline Variable & Total, $N(\%)$ \\
\hline $\begin{array}{l}\text { Neoadjuvant chemotherapy } \\
\text { No }\end{array}$ & $1061(92.6)$ \\
Yes & $85(7.4)$ \\
\hline$I Q R$ interquartile range, $A S A$ American Society of Anesthesiologist, \\
$C A$ carbohydrate antigen, $C E A$ carcinoembryonic antigen, $M F$ mass- \\
forming, IG intraductal growth, $P I$ periductal infiltrating, $A L B I$ \\
albumin-bilirubin, AJCC American Joint Committee on Cancer
\end{tabular}

Roughly one-third of patients received adjuvant chemotherapy $(n=342,31.3 \%)$, whereas only $7.4 \%$ $(n=85)$ received neoadjuvant chemotherapy (Table 1).

\section{Preoperative CART: Identification of Patients Who will Benefit the Most from SURGERY}

Among preoperative factors, CART analysis revealed that tumor number and size, ALBI grade and preoperative $\mathrm{LN}$ status were the strongest prognostic factors associated with OS among patients undergoing resection for ICC (Fig. 1). Of note, 5-year OS among patients with 1, 2-3, and $>3$ tumors undergoing resection was $42.1 \%, 27.5 \%$ and $3.8 \%$, respectively (Supplemental Figure 1a; $p<0.001)$. Among patients with a single tumor, CART selected tumor size, ALBI grade and preoperative LN status as the first, second, and third most important preoperative factors relative to OS. Of note, patients with an ICC $\leq 5 \mathrm{~cm}$ had a 5 -year OS of $52.1 \%$, whereas patients with larger tumors $(>5 \mathrm{~cm})$ had a 5 -year OS of only $34.4 \%$ (Supplemental Figure 1b; $p<0.001$ ). Among patients with small $(\leq 5 \mathrm{~cm})$ ICC, patients with ALBI grade 1 had better OS versus patients with ALBI grade 2/3 (57.9\% vs. $41 \%, p=0.008)$. Patients with all four favorable characteristics (i.e., single ICC, size $\leq 5 \mathrm{~cm}$, ALBI grade 1 and negative preoperative LN status) had a 5-year OS of $60.5 \%$. In contrast, 5-year OS decreased to $36.6 \%$ among patients who had 3 of 4 characteristics (i.e., single ICC, size $\leq 5 \mathrm{~cm}$, ALBI grade 1) and who had suspicious LNs based on preoperative assessment (Fig. 1). The model performed well in both the training (c-index: 0.74) and the validation dataset with bootstrapping resamples (c-index: $0.67)$.

\section{Determination of Preoperative Groups: Baseline Characteristics and Outcomes}

The preoperative CART model generated 4 groups of patients with distinct outcomes: Group $1(n=228)$ : single ICC, size $\leq 5 \mathrm{~cm}$, ALBI grade I, no preoperative LN metastasis; Group 3 ( $n=150)$ : 2-3 tumors; and Group 4 


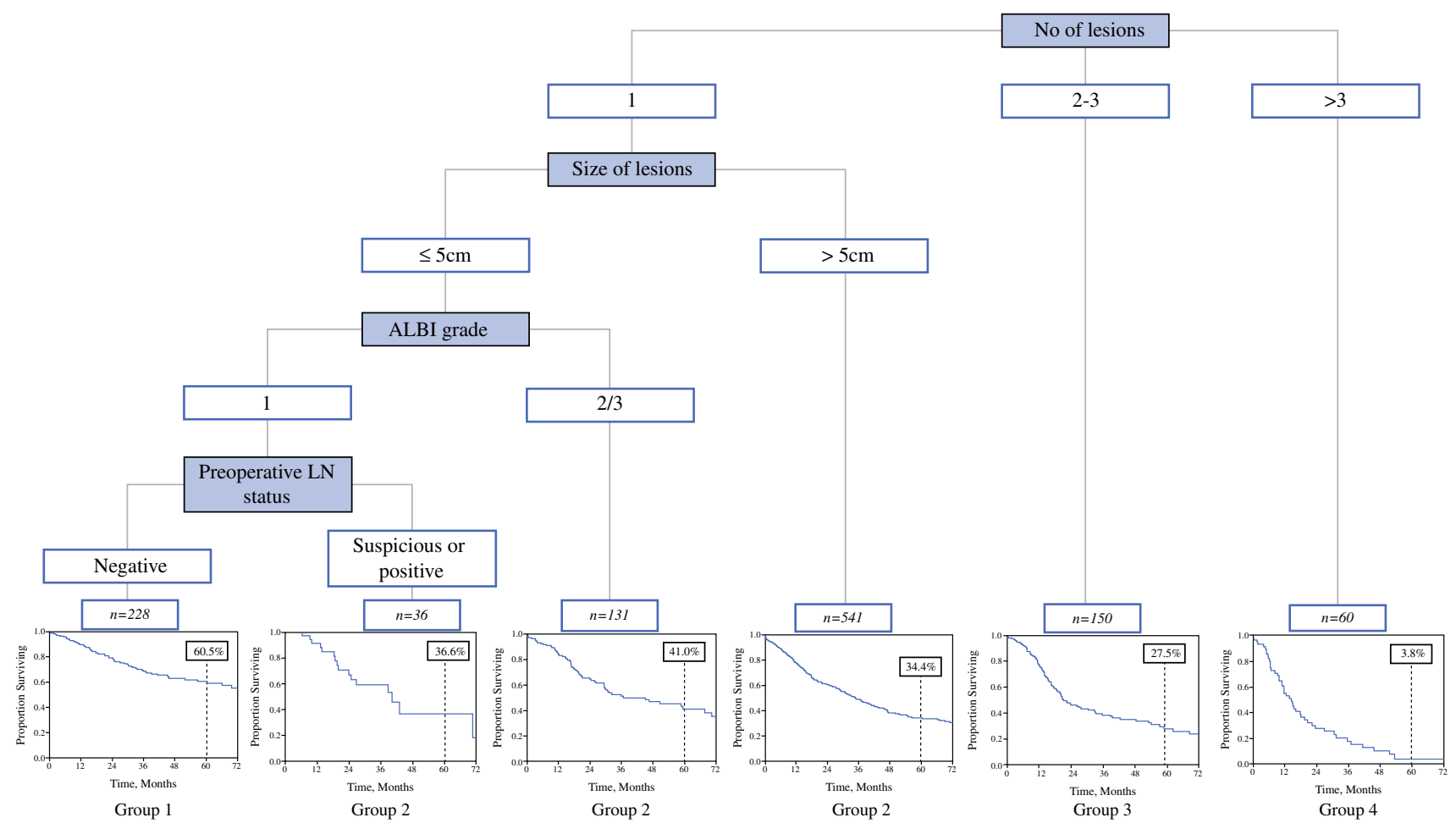

FIG. 1 Classification and Regression Tree (CART) model depicting the hierarchical association of preoperative factors relative to 5-year OS among patients who underwent surgery for ICC

( $n=60): \geq 4$ tumors. Of note, patients with either a single tumor $>5 \mathrm{~cm}$ or a single tumor $\leq 5 \mathrm{~cm}$, ALBI grade $2 / 3$ or a single tumor $\leq 5 \mathrm{~cm}$, ALBI grade 1 , metastatic/suspicious LNs all had a comparable long-term prognosis; in turn, these patients were categorized in a single cohort as Group 2 ( $n=708$; Fig. 1).

Differences in baseline characteristics among the four groups are summarized in Table 2. Of note, Group 1 patients had lower CA19-9 levels [Group 1: $26.9 \mathrm{UI} / \mathrm{mL}$ (IQR: 13-89.5), Group 2: $55.7 \mathrm{UI} / \mathrm{mL}$ (IQR: 19.2-236.7), Group 3: 76 UI/mL (IQR: 15.4-188.2), Group 4: 204 UI/ $\mathrm{mL}$ (IQR: 48-790), $p<0.001)]$ and lower CEA levels [Group 1: $2.2 \mathrm{ng} / \mathrm{L}$ (IQR: 1.4-3.3), Group 2: $2.5 \mathrm{ng} / \mathrm{L}$ (IQR: 1.5-4.6), Group 3: 2.2 ng/L (IQR: 1.2-4.3), Group 4: $2.9 \mathrm{ng} / \mathrm{L}$ (IQR: 1.7-6.5), $p=0.021)]$ compared with patients in Groups 2, 3, and 4 (Table 2). In addition, Group 1 patients more frequently had an R0 resection [Group 1: 212 (93.8\%), Group 2: 591 (84.2\%), Group 3: 129 (86.6\%), Group 4: $42(79.2 \%), p=0.006)]$, MF or IG ICC type [Group 1: 210 (94.6\%), Group 2: 566 (85.2\%), Group 3: $126(87.5 \%)$, Group 4: $33(71.7 \%), p<0.001)$ ], and no major vascular [Group 1: 205 (90.3\%), Group 2: 596 (85.1\%), Group 3: 131 (87.9\%), Group 4: 40 (75.5\%), $p=0.025)$ ] or microvascular invasion [Group 1: 172 (76.8\%), Group 2: 472 (67.7\%), Group 3: 95 (65.1\%), Group 4: $25(49.0 \%), p=0.001)]$ compared with the other CART groups (Table 2). Five-year OS among Groups 1, 2,
3 , and 4 patients was $60.5 \%, 35.8 \%, 27.5 \%$, and $3.8 \%$, respectively (Fig. 2a; $p<0.001$ ). Five-year DFS among Groups 1, 2, 3, and 4 patients was 47\%, 27.2\%, 6.8\%, and $0 \%$, respectively (Fig. $2 \mathrm{~b} ; p<0.001$ ). The preoperative CART model discriminated prognosis better than the 8 th edition of the AJCC T category designations (Fig. 2c) and performed comparably to the overall 8th edition of the AJCC staging system (Fig. 2d).

Among patients with multiple tumors (i.e., Groups 3 and 4 patients), patients with unilobar disease had a median and 5 -year OS of 20.9 months (95\% confidence interval [CI] 17.5-24.2) and $21.5 \%$, respectively, compared with 14.6 months (95\% CI 11.3-17.9) and $19.1 \%$ among patients with bilobar disease $(p=0.072)$. On multivariable analysis, CA19-9 $>200 \mathrm{UI} / \mathrm{mL}$ (hazard ratio $[\mathrm{HR}]=2.41$, 95\% CI 1.59-3.65, $p<0.001$ ), N1 status (HR $=1.66,95 \%$ CI $1.09-2.53, p=0.024), \mathrm{PI} / \mathrm{MF}+\mathrm{PI}$ ICC type $(\mathrm{HR}=$ $2.32,95 \%$ CI $1.45-3.69, p<0.001)$, as well as poor/undifferentiated tumor status (HR $=1.57,95 \%$ CI $1.08-2.28$, $p=0.017)$ were associated with worse survival among patients with multiple tumors (Table 3 ).

\section{DISCUSSION}

ICC is a rare cancer generally with an aggressive biologic behavior. ${ }^{1,4}$ Despite curative-intent resection, the prognosis of patients with ICC still remains dismal with 
TABLE 2 Differences in baseline characteristics between preoperative groups

\begin{tabular}{|c|c|c|c|c|c|}
\hline Variable & Group 1 & Group 2 & Group 3 & Group 4 & $p$ value \\
\hline Age, median (IQR) & $58(49,69)$ & $60(52,69)$ & $63(54,70.8)$ & $60.6(49.9,69.3)$ & 0.15 \\
\hline Male & $153(67.1)$ & $376(53.2)$ & $76(50.7)$ & $32(53.3)$ & 0.001 \\
\hline ASA & & & & & $<0.001$ \\
\hline$\leq 2$ & $158(80.6)$ & $388(66.6)$ & $66(54.1)$ & $20(46.5)$ & \\
\hline$>2$ & $38(19.4)$ & $195(33.4)$ & $56(45.9)$ & $23(53.5)$ & \\
\hline CA19-9, UI/mL, median (IQR) & $26.9(13,89.5)$ & $55.7(19.2,236.7)$ & $76(15.4,188.2)$ & $204(48,790)$ & $<0.001$ \\
\hline CEA, ng/L, median (IQR) & $2.2(1.4,3.3)$ & $2.5(1.5,4.6)$ & $2.2(1.2,4.3)$ & $2.9(1.7,6.5)$ & 0.021 \\
\hline ALBI grade & & & & & $<0.001$ \\
\hline 1 & $170(100)$ & $263(53.9)$ & $42(57.5)$ & $12(41.4)$ & \\
\hline $2 / 3$ & - & $225(46.1)$ & $31(42.5)$ & $17(58.6)$ & \\
\hline Cirrhosis & $43(18.9)$ & $61(8.7)$ & $11(7.3)$ & $8(13.8)$ & $<0.001$ \\
\hline Type of resection & & & & & $<0.001$ \\
\hline Minor resection & $129(61.1)$ & $226(35.2)$ & $35(27.1)$ & $12(26.1)$ & \\
\hline Major resection & $82(38.9)$ & $416(64.8)$ & $94(72.9)$ & $34(73.9)$ & \\
\hline Preoperative LN status & & & & & $<0.001$ \\
\hline Negative & $196(100)$ & $420(73.0)$ & $79(71.8)$ & $25(69.4)$ & \\
\hline Suspicious & - & $109(19.0)$ & $23(20.9)$ & $5(13.9)$ & \\
\hline Positive & - & $46(8.0)$ & $8(7.3)$ & $6(16.7)$ & \\
\hline Tumor size $(\mathrm{cm})$, median (IQR) & $3.6(2.5,4.5)$ & $7(5.2,9.0)$ & $7(5.0,9.0)$ & $7(5.0,10.0)$ & $<0.001$ \\
\hline Margin status & & & & & 0.006 \\
\hline R0 & $212(93.8)$ & $591(84.2)$ & $129(86.6)$ & $42(79.2)$ & \\
\hline $\mathrm{R} 1 / \mathrm{R} 2$ & $14(6.2)$ & $111(15.8)$ & $20(13.4)$ & $11(20.8)$ & \\
\hline Morphologic type & & & & & $<0.001$ \\
\hline MF, IG & $210(94.6)$ & $566(85.2)$ & $126(87.5)$ & $33(71.7)$ & \\
\hline $\mathrm{PI}, \mathrm{MF}+\mathrm{PI}$ & $12(5.4)$ & $98(14.8)$ & $18(12.5)$ & $13(28.3)$ & \\
\hline Grade & & & & & $<0.001$ \\
\hline Well to moderate & $194(90.7)$ & $547(82.6)$ & $103(73.0)$ & $34(69.4)$ & \\
\hline Poor to undifferentiated & $20(9.3)$ & $115(17.4)$ & $38(27.0)$ & $15(30.6)$ & \\
\hline Major vascular invasion & & & & & 0.025 \\
\hline No & $205(90.3)$ & $596(85.1)$ & $131(87.9)$ & $40(75.5)$ & \\
\hline Yes & $22(9.7)$ & $104(14.9)$ & $18(12.1)$ & $13(24.5)$ & \\
\hline Microvascular invasion & & & & & 0.001 \\
\hline No & $172(76.8)$ & $472(67.7)$ & $95(65.1)$ & $25(49.0)$ & \\
\hline Yes & $52(23.2)$ & $225(32.3)$ & $51(34.9)$ & $26(51.0)$ & \\
\hline Median OS, months (95\% CI) & $79.2(\mathrm{NR})$ & $36.2(31.2,41.1)$ & $21.2(15.7,26.8)$ & $14.6(10.3,18.9)$ & \\
\hline 5 -year OS $(\%)$ & $60.5 \%$ & $35.8 \%$ & $27.5 \%$ & $3.8 \%$ & \\
\hline
\end{tabular}

$I Q R$ interquartile range, ASA American Society of Anesthesiologist, $C A$ carbohydrate antigen, $C E A$ carcinoembryonic antigen, $M F$ massforming, $I G$ intraductal growth, $P I$ periductal infiltrating, $A L B I$ albumin-bilirubin, $N R$ not reached

many patients experiencing a recurrence within 2 years and less than $40 \%$ of patients being alive at 5 -years. ${ }^{3,4,6}$ In turn, accurate prediction of outcomes and identification of patients who will derive the most benefit from surgery has particular importance among patients with ICC. The current study was important, because we used a machinebased approach to identify subsets of patients with distinct prognosis using preoperative data derived from a CART model. The CART model facilitated a hierarchical weighting of the different prognostic factors to identify which preoperative factors held the most prognostic impact. Specifically, the CART model identified tumor number and size, ALBI grade and preoperative LN status as the most important factors to consider in the preoperative setting. The model identified six nodal points that translated into four distinct prognostic groups (some nodes 

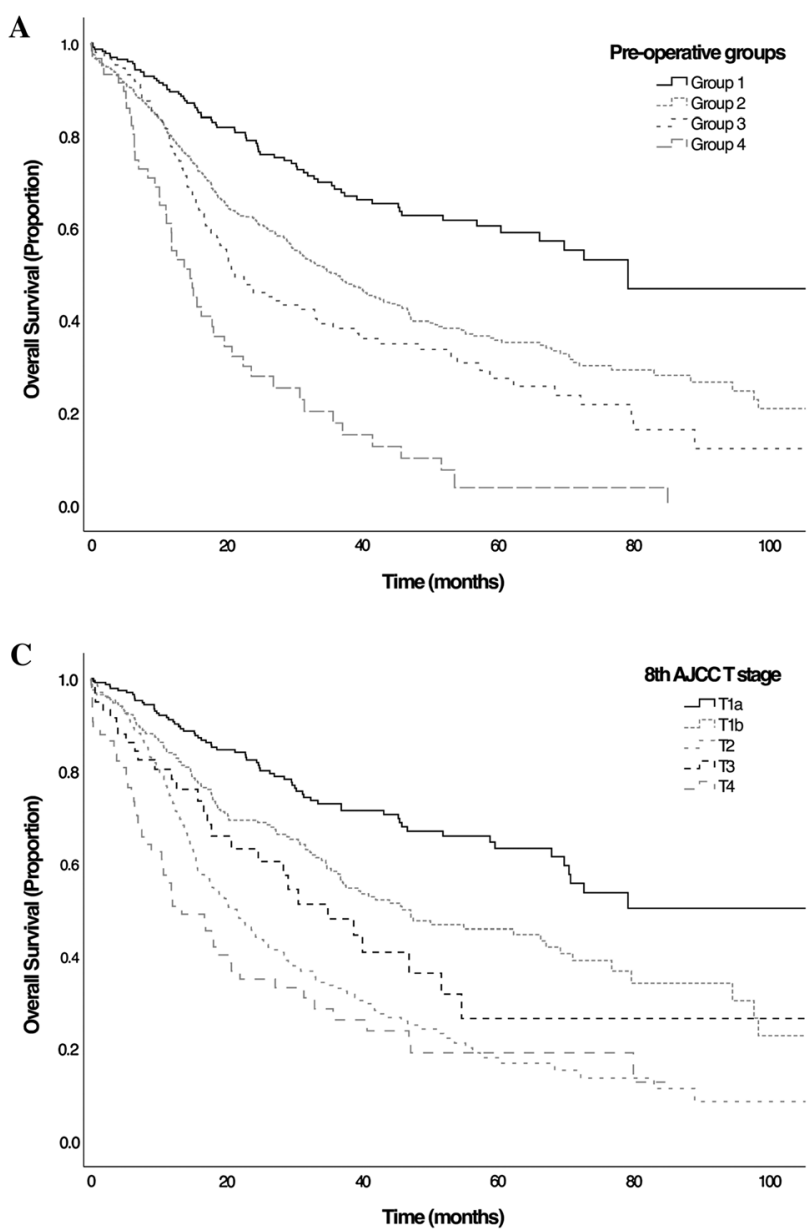

FIG. 2 Kaplan-Meier curve demonstrating differences in OS (a) and DFS (b) among patients of different preoperative groups. Although the CART model identified group of patients with incrementally worse OS, there was poor separation of survival curves among

were merged due to similar outcomes). Using the CART model, prognostic cohorts that had incrementally worse survival were defined. For example, patients in Group 1 (i.e., patients with all 4 favorable characteristics; single ICC, size $\leq 5 \mathrm{~cm}$, ALBI grade I, negative preoperative LN status) had a 5-year OS of $60.5 \%$, whereas patients in Group 4 (i.e., patients with $\geq 4$ tumors) had an abysmal 5 -year OS of only $3.8 \%$. To the best of our knowledge, this is the first study to utilize a machine-based method as a means to preoperatively identify different prognostic groups among ICC patients. In turn, these data may help to inform presurgical decisions, such as the preferential routine use of neoadjuvant therapy for patients in prognostic Group 4.

Machine learning techniques have recently gained popularity as a means to analyze large volumes of data to identify patterns and trends that may not be apparent to a human or evident with other statistical techniques. In general, machine learning approaches require minimal human intervention, thus limiting bias and error. ${ }^{14,15}$
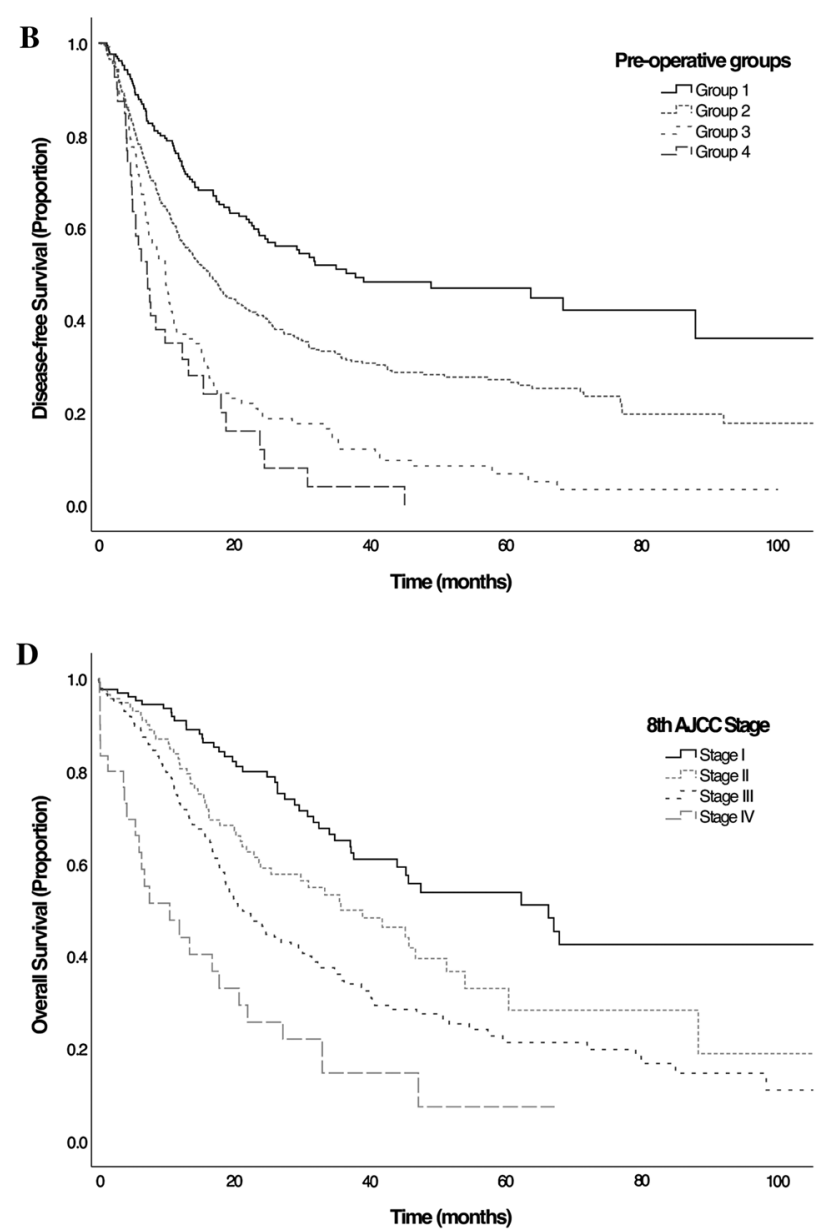

patients with different $\mathrm{T}$ stage disease (c). In contrast, the CART model could discriminate prognosis well and similarly to the overall 8th edition of the AJCC staging system (d)

CART analysis is a machine based technique that can account for complex relationships compared with traditional statistical methods. ${ }^{22}$ In particular, CART models may be helpful decision-making tools and have demonstrated promise in the field of surgery. ${ }^{14,15,23}$ For example, among patients with aggressive malignancies, such as ICC, CART models may be particularly useful in the selection of patients for surgery by identifying those individuals who might benefit more from going directly to surgery versus being treated with other options, such as neoadjuvant chemotherapy or locoregional modalities. In addition, although nomograms have previously been published to predict outcomes among patients undergoing partial hepatectomy for ICC, these have largely been based on data derived from the postoperative period (e.g., vascular invasion, LN metastasis, pathologic tumor size, number, etc.) and, thus, cannot be applied to patients in the preoperative setting. ${ }^{10}$ In the current study, the CART model used purely preoperative factors and identified tumor size and number as the first and second most important 
TABLE 3 Factors associated with survival among patients with multifocal tumors $(n=210)$

\begin{tabular}{|c|c|c|c|c|}
\hline \multirow[t]{2}{*}{ Variable } & \multicolumn{2}{|l|}{ Bivariate } & \multicolumn{2}{|l|}{ Multivariable } \\
\hline & HR (95\% CI) & $p$ value & $\mathrm{HR}(95 \% \mathrm{CI})$ & $p$ value \\
\hline Age & $0.99(0.98-1.01)$ & 0.38 & - & - \\
\hline \multicolumn{5}{|l|}{ Gender } \\
\hline Male & Ref & & & \\
\hline Female & $0.83(0.59-1.16)$ & 0.83 & - & - \\
\hline \multicolumn{5}{|l|}{ ASA } \\
\hline$\leq 2$ & Ref & & & \\
\hline$>2$ & $1.13(0.78-1.65)$ & 0.52 & - & - \\
\hline \multicolumn{5}{|l|}{ CA19-9, UI/mL } \\
\hline$\leq 200$ & Ref & & Ref & \\
\hline$>200$ & $3.08(2.17-4.37)$ & $<0.001$ & $2.41(1.59-3.65)$ & $<0.001$ \\
\hline \multicolumn{5}{|l|}{ CEA, ng/L } \\
\hline$\leq 5$ & Ref & & Ref & \\
\hline$>5$ & $2.33(1.65-3.30)$ & $<0.001$ & $1.45(0.96-2.18)$ & 0.075 \\
\hline \multicolumn{5}{|l|}{ Cirrhosis } \\
\hline No & Ref & & & \\
\hline Yes & $1.41(0.78-2.54)$ & 0.25 & - & - \\
\hline \multicolumn{5}{|l|}{ ALBI grade } \\
\hline 1 & Ref & & & \\
\hline $2 / 3$ & $1.45(0.89-2.37)$ & 0.14 & - & - \\
\hline \multicolumn{5}{|l|}{ Location } \\
\hline Unifocal & Ref & & & \\
\hline Multifocal & $1.42(0.97-2.09)$ & 0.07 & - & - \\
\hline Tumor size & $1.04(0.99-1.09)$ & 0.10 & - & - \\
\hline Tumor number & $1.21(1.11-1.32)$ & $<0.001$ & $1.03(0.95-1.12)$ & 0.39 \\
\hline \multicolumn{5}{|c|}{ AJCC 8th edition $\mathrm{N}$ stage } \\
\hline No & Ref & & Ref & \\
\hline N1 & $1.97(1.20-3.25)$ & 0.007 & $1.66(1.09-2.53)$ & 0.024 \\
\hline $\mathrm{Nx}$ & $1.25(0.80-1.96)$ & 0.33 & $1.38(0.86-2.22)$ & 0.18 \\
\hline \multicolumn{5}{|l|}{ Margin status } \\
\hline R0 & Ref & & Ref & \\
\hline $\mathrm{R} 1 / \mathrm{R} 2$ & $1.86(1.13-3.05)$ & 0.014 & $1.41(0.83-2.41)$ & 0.20 \\
\hline \multicolumn{5}{|l|}{ Morphologic type } \\
\hline MF, IG & Ref & & Ref & \\
\hline $\mathrm{PI}, \mathrm{MF}+\mathrm{PI}$ & $2.98(1.92-4.64)$ & $<0.001$ & $2.32(1.45-3.69)$ & $<0.001$ \\
\hline \multicolumn{5}{|l|}{ Grade } \\
\hline Well/moderate & Ref & & Ref & \\
\hline Poor/undifferentiated & $1.74(1.20-2.52)$ & 0.004 & $1.57(1.08-2.28)$ & 0.017 \\
\hline \multicolumn{5}{|l|}{ Major vascular invasion } \\
\hline No & Ref & & & \\
\hline Yes & $1.33(0.85-2.09)$ & 0.22 & - & - \\
\hline \multicolumn{5}{|l|}{ Microvascular invasion } \\
\hline No & Ref & & & \\
\hline Yes & $1.17(0.82-1.66)$ & 0.39 & - & - \\
\hline \multicolumn{5}{|l|}{ Adjuvant chemotherapy } \\
\hline No & Ref & & & \\
\hline Yes & $0.88(0.62-1.25)$ & 0.48 & - & - \\
\hline
\end{tabular}

Bold values represent statistical significance

ASA American Society of Anesthesiologist, $C A$ Carbohydrate antigen, $C E A$ carcinoembryonic antigen, $M F$ mass-forming, $I G$ intraductal growth, $P I$ periductal infiltrating, $A L B I$ albumin-bilirubin, AJCC American Joint Committee on Cancer 
preoperative factors associated with prognosis. Of note, 5 -year OS incrementally worsened with increasing number of tumors reaching a 5-year OS of less than 5\% among patients with $>3$ tumors $(1,2-3$, and $>3$ tumors: 5 -year OS: $42.1 \%, 27.5 \%$, and $3.8 \%, p<0.001$; Supplemental Figure 1a). Addeo et al. ${ }^{24}$ had similarly reported a very low 5 -year OS of $7 \%$ among patients with multinodular ICC. Although the 8th edition AJCC staging manual considers multinodular ICC as a single category (T2 stage) regardless of the number of tumors, the current study noted that prognosis incrementally worsened as the actual number of lesions increased. In examining patients with multiple tumors, patients with unilobar disease tended to have a longer median survival compared with individuals who had bilobar disease. Of note, high CA19-9 levels was particularly associated with a worse survival among patients with multiple tumors. As such, the data suggest that patients with multiple tumors-especially those with bilobar disease and high CA19-9 levels-identified in the preoperative setting should be strongly considered for neoadjuvant systemic or locoregional options before contemplating resection. ${ }^{24-28}$

Following tumor number, the next variable identified as being most important relative to prognosis in the CART model was tumor size. In fact, among patients with a solitary tumor, tumor size was able to stratify prognosis of patients based on an ICC tumor size cutoff of $>5 \mathrm{~cm}$ $(\leq 5 \mathrm{~cm} 52.1 \%$ vs. $>5 \mathrm{~cm}$ ICC $34.4 \%, p<0.001)$. These data were consistent with the latest revision of the 8th edition of the AJCC staging manual. ${ }^{16}$ The importance of tumor size in the prognosis of patients with ICC has been somewhat controversial as the 7th edition of the AJCC staging manual did not include tumor size. ${ }^{29,30}$ Subsequent data suggested, however, that tumor size was indeed prognostically important. As such, the 8th edition AJCC staging manual now has two $\mathrm{T}$ stage categories for solitary ICC based on tumor size (single $\leq 5 \mathrm{~cm}$ (T1a) and $>5 \mathrm{~cm}$ (T1b)) 9,16,29 The CART analysis indeed confirmed the relative importance of tumor size. However, the effect of tumor size was somewhat mediated by ALBI grade. Specifically, among patients with single ICC $\leq 5 \mathrm{~cm}$ (T1a), survival outcomes varied considerably with ALBI grade, which was the next most important predictor of outcomes in the CART model. In fact, patients with ICC $\leq 5 \mathrm{~cm}$ and ALBI grade 1 had a better OS versus patients with ALBI grade $2 / 3$ (57.9\% vs. $41 \%, p=0.008)$. While the reason for this is likely multifactorial, a higher ALBI grade likely acted as a surrogate for liver function reserve. ${ }^{12,31}$ Collectively, these data highlight the importance of the preoperative assessment of liver parenchymal quality and underlying liver function even among patients with small $(\leq 5 \mathrm{~cm})$ solitary tumors prior to surgery for ICC..$^{32}$ In addition to assessment of liver function, appraisal of LN status was also important. To this point, patients with single ICC, size $\leq 5 \mathrm{~cm}$, ALBI grade 1 with no malignant LN identified on preoperative imaging had a 5 -year OS as high as $60.5 \%$. In contrast, patients with the same favorable characteristics, but who had suspicious or metastatic LNs identified preoperatively had roughly onehalf the 5-year OS (Fig. 1). Collectively, the CART model allowed for the identification of four groups of patients with distinct outcomes following resection for ICC (Table 2). The clinician simply can follow the paths of the tree that best describe the characteristics of the patient being evaluated and can arrive at the prediction of the outcome of interest for that particular patient.

The current study had several limitations that should be considered when interpreting the results. As with all retrospective studies, the current study was subject to selection bias. In addition, although the analysis of data from multiple centers was a strength, there might have been some heterogeneity in patient selection and surgical techniques among the different participating centers. Although the large number of ICC patients in the cohort was a strength, the long study period (i.e., 1990-2017) may have been a source of bias since techniques and perioperative care for ICC patients have changed over the years. Patients were, however, relatively equally distributed over the years of the study period. Furthermore, variations may also exist in the evaluation of tumor size and number in different centers depending on the method of assessment and operator expertise. Previous data would suggest, however, that these differences were unlikely to be clinically significant. ${ }^{33}$

\section{CONCLUSIONS}

Surgery was associated with dismal prognosis among certain patients with ICC. Tumor size and number were the strongest determinants of outcomes prior to surgery and should guide patient selection. Assessment of liver function reserve as well as evaluation of preoperative LN status should also be considered when examining surgery for ICC patients. In turn, the CART model was able to stratify patients in 4 groups with a 5-year OS ranging from 60.5 to $3.8 \%$. Patients with multiple tumors, especially those with bilobar disease and high CA19-9 levels, identified in the preoperative setting should be strongly considered for neoadjuvant systemic or locoregional options before contemplating resection. The machine-based, CART model could provide an easy to interpret representation of variable outcomes relative to preoperative factors and could be used as a guide for preoperative patient selection and risk stratification. 
FUNDING None.

\section{REFERENCES}

1. Wu L, Tsilimigras DI, Paredes AZ, et al. Trends in the incidence, treatment and outcomes of patients with intrahepatic cholangiocarcinoma in the USA: facility type is associated with margin status, use of lymphadenectomy and overall survival. World $J$ Surg. 2019; 43:1777-87.

2. Singal AK, Vauthey JN, Grady JJ, Stroehlein JR. Intra-hepatic cholangiocarcinoma-frequency and demographic patterns: thirtyyear data from the M.D. Anderson Cancer Center. J Cancer Res Clin Oncol. 2011;137(7):1071-8.

3. de Jong MC, Nathan H, Sotiropoulos GC, et al. Intrahepatic cholangiocarcinoma: an international multi-institutional analysis of prognostic factors and lymph node assessment. J Clin Oncol. 2011;29(23):3140-5.

4. Endo I, Gonen M, Yopp AC, et al. Intrahepatic cholangiocarcinoma: rising frequency, improved survival, and determinants of outcome after resection. Ann Surg. 2008;248(1):84-96.

5. Merath K, Chen Q, Bagante F, et al. A Multi-institutional International Analysis of Textbook Outcomes Among Patients Undergoing Curative-Intent Resection of Intrahepatic Cholangiocarcinoma. JAMA Surg. 2019;154(6):e190571.

6. Zhang XF, Beal EW, Bagante F, et al. Early versus late recurrence of intrahepatic cholangiocarcinoma after resection with curative intent. Br J Surg. 2018;105(7):848-56.

7. Yadav S, Xie H, Bin-Riaz I, et al. Neoadjuvant vs. adjuvant chemotherapy for cholangiocarcinoma: a propensity score matched analysis. Eur J Surg Oncol. 2019;45(8):1432-8.

8. Buettner S, Galjart B, van Vugt JLA, et al. Performance of prognostic scores and staging systems in predicting long-term survival outcomes after surgery for intrahepatic cholangiocarcinoma. J Surg Oncol. 2017;116(8):1085-95.

9. Hyder O, Marques $\mathrm{H}$, Pulitano C, et al. A nomogram to predict long-term survival after resection for intrahepatic cholangiocarcinoma: an Eastern and Western experience. JAMA Surg. 2014;149(5):432-8.

10. Wang Y, Li J, Xia Y, et al. Prognostic nomogram for intrahepatic cholangiocarcinoma after partial hepatectomy. Journal of clinical oncology: official journal of the American Society of Clinical Oncology. 2013;31(9):1188-95.

11. Sasaki K, Margonis GA, Andreatos N, et al. Preoperative Risk Score and Prediction of Long-Term Outcomes after Hepatectomy for Intrahepatic Cholangiocarcinoma. $J$ Am Coll Surg. 2018;226(4):393-403.

12. Tsilimigras DI, Hyer JM, Moris D, et al. Prognostic utility of albumin-bilirubin grade for short- and long-term outcomes following hepatic resection for intrahepatic cholangiocarcinoma: a multi-institutional analysis of 706 patients. J Surg Oncol. 2019;120(2):206-213.

13. Moro A, Paredes AZ, Farooq A, et al. Discordance in prediction of prognosis among patients with intrahepatic cholangiocarcinoma: a preoperative vs postoperative perspective. J Surg Oncol. 2019;120(6):946-955.

14. Bagante F, Spolverato G, Merath K, et al. Intrahepatic cholangiocarcinoma tumor burden: a classification and regression tree model to define prognostic groups after resection. Surgery. 2019. https://doi.org/10.1016/j.surg.2019.06.005.

15. Wiener M, Acland KM, Shaw HM, et al. Sentinel node positive melanoma patients: prediction and prognostic significance of nonsentinel node metastases and development of a survival tree model. Ann Surg Oncol. 2010;17(8):1995-2005.

16. Amin MB, Greene FL, Edge SB, et al. The Eighth Edition AJCC Cancer Staging Manual: Continuing to build a bridge from a population-based to a more "personalized" approach to cancer staging. CA Cancer J Clin. 2017;67(2):93-9

17. Strasberg SM. Nomenclature of hepatic anatomy and resections: a review of the Brisbane 2000 system. J Hepatobiliary Pancreat Surg. 2005;12(5):351-5.

18. Johnson PJ, Berhane S, Kagebayashi C, et al. Assessment of liver function in patients with hepatocellular carcinoma: a new evidence-based approach-the ALBI grade. $J$ Clin Oncol. 2015;33(6):550-8.

19. Breiman L. (1984) Classification and regression trees. Belmont: Wadsworth International Group.

20. Zeileis A, Hothorn T. Partykit: a toolkit for recursive partytioning. https://cran.r-project.org/web/packages/partykit/vignettes/pa rtykit.pdf.

21. Intrator $\mathrm{O}$, Kooperberg $\mathrm{C}$. Trees and splines in survival analysis. Stat Methods Med Res. 1995;4(3):237-61.

22. Valera VA, Walter BA, Yokoyama N, et al. Prognostic groups in colorectal carcinoma patients based on tumor cell proliferation and classification and regression tree (CART) survival analysis. Ann Surg Oncol. 2007;14(1):34-40.

23. Hyer JM, Ejaz A, Tsilimigras DI, Paredes AZ, Mehta R, Pawlik TM. Novel machine learning approach to identify preoperative risk factors associated with super-utilization of medicare expenditure following surgery. JAMA Surg. 2019. https://doi.org/10. 1001/jamasurg.2019.2979.

24. Addeo P, Jedidi I, Locicero A, et al. Prognostic impact of tumor multinodularity in intrahepatic cholangiocarcinoma. J Gastrointest Surg. 2018; 23:1801-1809.

25. Sahara K, Tsilimigras DI, Mehta R, et al. A novel online prognostic tool to predict long-term survival after liver resection for intrahepatic cholangiocarcinoma: the "metro-ticket" paradigm. $J$ Surg Oncol. 2019;120(2):223-30.

26. Reames BN, Bagante F, Ejaz A, et al. Impact of adjuvant chemotherapy on survival in patients with intrahepatic cholangiocarcinoma: a multi-institutional analysis. HPB (Oxford). 2017;19(10):901-9.

27. Buettner S, Koerkamp BG, Ejaz A, et al. The effect of preoperative chemotherapy treatment in surgically treated intrahepatic cholangiocarcinoma patients: a multi-institutional analysis. $J$ Surg Oncol. 2017;115(3):312-8.

28. Hyder O, Marsh JW, Salem R, et al. Intra-arterial therapy for advanced intrahepatic cholangiocarcinoma: a multi-institutional analysis. Ann Surg Oncol. 2013;20(12):3779-86.

29. Kim Y, Moris DP, Zhang XF, et al. Evaluation of the 8th edition American Joint Commission on Cancer (AJCC) staging system for patients with intrahepatic cholangiocarcinoma: a surveillance, epidemiology, and end results (SEER) analysis. J Surg Oncol. 2017;116(6):643-50.

30. Spolverato G, Bagante F, Weiss M, et al. Comparative performances of the 7 th and the 8th editions of the American Joint Committee on Cancer staging systems for intrahepatic cholangiocarcinoma. J Surg Oncol. 2017;115(6):696-703.

31. Pinato DJ, Sharma R, Allara E, et al. The ALBI grade provides objective hepatic reserve estimation across each BCLC stage of hepatocellular carcinoma. J Hepatol. 2017;66(2):338-46.

32. Squires MH, Cloyd JM, Dillhoff M, Schmidt C, Pawlik TM. Challenges of surgical management of intrahepatic cholangiocarcinoma. Exp Rev Gastroenterol Hepatol. 2018;12(7):671-81.

33. Chan AWH, Zhong J, Berhane S, et al. Development of pre and post-operative models to predict early recurrence of hepatocellular carcinoma after surgical resection. $J$ Hepatol. 2018;69(6):1284-93.

Publisher's Note Springer Nature remains neutral with regard to jurisdictional claims in published maps and institutional affiliations. 